\title{
An attempt to evaluate intraductal components of breast cancer by dual energy computed tomography
}

- Objectives: Evaluation of Intraductal Components (ICS) of breast cancer, such as ductal extension or segmental distribution, is essential before surgery. Evaluation is mainly performed using contrast-enhanced MRI as its sensitivity and detectability are superior to those of contrast-enhanced CT. Dual Energy CT (DECT) has been shown to have a high contrast enhancement effect with high resolution in soft tissues such as breast tissue. Thus, we assessed the utility of DECT for evaluating an IC of breast cancer.

- Methods: Fifty-five patients with a biopsy-proven breast cancer have undergone contrast-enhanced DECT and MRI for preoperative investigation. We compared the imaging findings of both modalities and correlated them with the pathological outcome.

- Results: Pathologically, an IC of breast cancer was identified in 26 patients. Existence of an IC was identified in 32 patients on DECT and 35 patients on MRI. The sensitivity, specificity and accuracy for detecting an IC were $76.9 \%, 58.6 \%$ and $67.3 \%$ with DECT and were $73.1 \%, 44.8 \%$, and $58.2 \%$ with MRI, respectively.

- Conclusions: The appearance of ICs on DECT was highly correlated with the pathological outcome. The advantages of DECT include high detectability of an IC, clinically acceptable image quality, imaging in the face-up position (consistent with the surgical position) and simultaneous assessment of distant metastasis without radiation dose penalty. Thus, DECT is a useful supporting modality for the detection of an IC of breast cancer.

KEYWORDS: dual-energy CT - breast cancer - intraductal component - extensive intraductal component - ductal extension - contrast-enhanced CT - radiation dose penalty

\section{Introduction}

Surgery is a common treatment approach for breast cancer, and most patients with breast cancer undergo some type of surgery as part of treatment $[1,2]$. Assessment of the main tumour and evaluation of the presence of Intraductal Components (ICs) are essential before surgery to determine the most appropriate surgical procedure. A positive surgical margin is mainly the result of inadequate resection of a cancer's IC $[3,4]$. Thus, precise pre-operative assessment of the presence and range of an IC is extremely important to achieve a cancer-free surgical margin. Several early studies with MRI imaging of the breast cancer were aimed for evaluation of the presence of extensive intraductal component (EIC) [5-7]. EICwas defined as Ductal Carcinoma In Situ, (DCIS) occupying $25 \%$ or more of the primary tumour with intraductal foci [8-10]. In MRI inspection, EIC is demonstrated as spotty or linear continuous enhancement from the main tumour to the nipple [5-10]. Shimauchi et al. assessed both of EIC and DCIS without invasive ductal carcinoma as ICs and classified them into ductal extension type and segmental distribution type [11]. In this study, we also assessed both of EIC and DCIS without invasive ductal carcinoma as ICs in both of pathological and imaging inspection.

MRI has shown high sensitivity and high detectability of ICs compared with different modalities such as Single-Energy Computed Tomography (SECT) [12-14]. Contrastenhanced SECT is mainly used to estimate the presence of metastatic tumours and regional lymphadenopathy, as an IC of breast cancer is a fine structure with attenuation similar to that of normal breast tissue and is difficult to distinguish in SECT images. Dual-Energy CT (DECT) is an advancement in CT technology, and it has been shown to have potential for the detection and characterisation of abdominal and pulmonary diseases [15-23]. Additionally, DECT images reconstructed at low photon energies have a high contrast enhancement effect with high resolution in soft tissues $[17,22,23]$. These findings suggest the potential of DECT for detecting fine structures, such as an IC of breast cancer, in surrounding normal breast tissue. However, the utility of contrastenhanced DECT for visualizing and evaluating an IC of breast cancer is unclear. Thus, the present study aimed to evaluate the utility of contrast-enhanced DECT for visualizing and

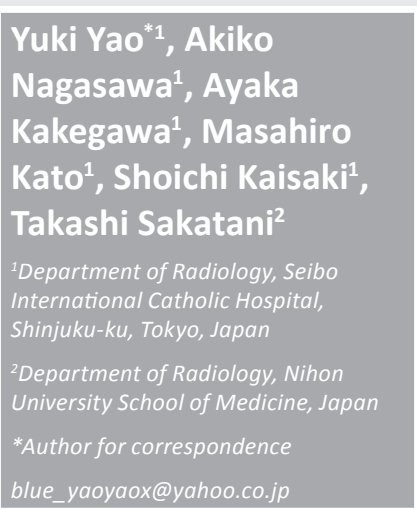


evaluating an IC of breast cancer. Some previous studies have focused on DECT examination of breast tissue or breast cancer [24-29], and it is expected that the findings of the present study will encourage further advancement in DECT imaging and will help improve visualization of an IC in patients with breast cancer.

\section{Materials and Methods - Patient selection}

The study was approved by our institutional review board, and the requirement for informed consent was waived owing to its retrospective design. The study included 55 patients (mean age, $55.3 \pm 18.1$ years; range $35-82$ years) with pathologically proven breast cancer on core needle biopsy between January 2015 and April 2017. Of the 55 patients, 7 had ductal carcinoma in situ, 29 had invasive breast cancer and 19 had non-invasive breast cancer. Patients who underwent previous chemotherapy or hormone therapy were excluded from this study. All 55 patients underwent MRI and DECT for pre-operative examination. The primary tumour and presence of an IC were assessed, and staging was performed.

\section{- Imaging examinations}

All patients underwent contrast-enhanced DECT and contrast-enhanced MRI as presurgical imaging examinations. DECT was mainly performed for pre-operative staging, and the presence of an IC was assessed simultaneously in all patients. MRI was subsequently performed to evaluate the spread of the primary tumour, the presence and range of an IC and the presence of other tumours in both breasts.

All CT examinations were performed using a third-generation DECT system (Multislice CT Scanner LightSpeed, Discovery CT750 HD with GSI configuration, GE Healthcare, Chicago, IL, USA). The system utilizes a single source and single detector geometry with rapid switching of tube $\mathrm{kV}$. In all patients, portal venous images were acquired $80 \mathrm{~s}$ after contrast enhancement, with a scan range from the chest to the inguinal region. The rotation time was 0.6 or $0.8 \mathrm{~s}$, and the tube current was 375 or $500 \mathrm{~mA}$. Automated attenuation-based tube voltage selection and tube current modulation techniques were applied (TABLE 1). Contrast attenuation was achieved using a body weightadapted protocol for the injection of $0.5 \mathrm{gI} /$ $\mathrm{kg}$ of non-ionic iodinated contrast material (Omnipaque 300, Daiichi Sankyo Healthcare, Co., Ltd, Tokyo, Japan) for $50 \mathrm{~s}$ via an automated injector. Images were reconstructed using a monochromatic algorithm with photon energies of 40 and $65 \mathrm{keV}$ (substitution for $120 \mathrm{kV}$ images) on a workstation (Advantage Workstation, GE Healthcare). Reconstructed $65 \mathrm{keV}$ images with a slice thickness of $5 \mathrm{~mm}$ were utilized to assess regional and distant metastasis, whereas $40 \mathrm{keV}$ images were utilized to investigate an IC and evaluate the detailed shape and extent of the tumour (FIGURES 1A and 2A). Reconstructed Maximum Intensity Projection (MIP) and Virtual Rendering (VR) images were also obtained as necessary (FIGURES 1C and 2C).

All images obtained were evaluated by two expert radiologists (each with more than 8 years of experience in evaluating an IC of breast cancer). The decision of IC presence was focused on the existence of ductal extension and segmental distribution regardless of their detailed size or shape. We compared the imaging findings of both modalities and correlated the findings with the pathological outcomes (FIGURES 2D and $2 \mathrm{E})$.

\section{- Image and statistical analyses}

For DECT, IC was evaluated in $40 \mathrm{keV}$ axial images with a slice thickness of $1 \mathrm{~mm}$. Multiplanar reformation images, 3D-MIP images or VR images were reconstructed as necessary. Metastatic tumour assessment and

\begin{tabular}{|cc|}
\hline \multicolumn{2}{|c|}{ Table 1. DECT protocol of data acquisition and image reconstruction. } \\
\hline Tube voltage (kV) & $\begin{array}{c}100: \text { automated Attenuation-based Tube Voltage Selection (ATVS) } \\
\text { and tube current modulation techniques }\end{array}$ \\
\hline Tube current $(\mathbf{m A})$ / Rotation time (sec) & $375 / 0.6$ (Medium FOV) \\
& $550 / 0.8$ (Large FOV) \\
\hline Detector configuration $(\mathbf{m m})$ & $0.625(\mathrm{x} 64)$ \\
\hline Pitch factor & 0.9 \\
\hline $\begin{array}{c}\text { Reconstruction parameter: } \\
\text { Slice }(\mathbf{m m}) / \text { Increment }(\mathbf{m m})\end{array}$ & $5 / 5(65 \mathrm{keV})$ \\
\hline Field of view $(\mathbf{m m})$ & $1 / 1(40,65 \mathrm{keV})$ \\
\hline
\end{tabular}



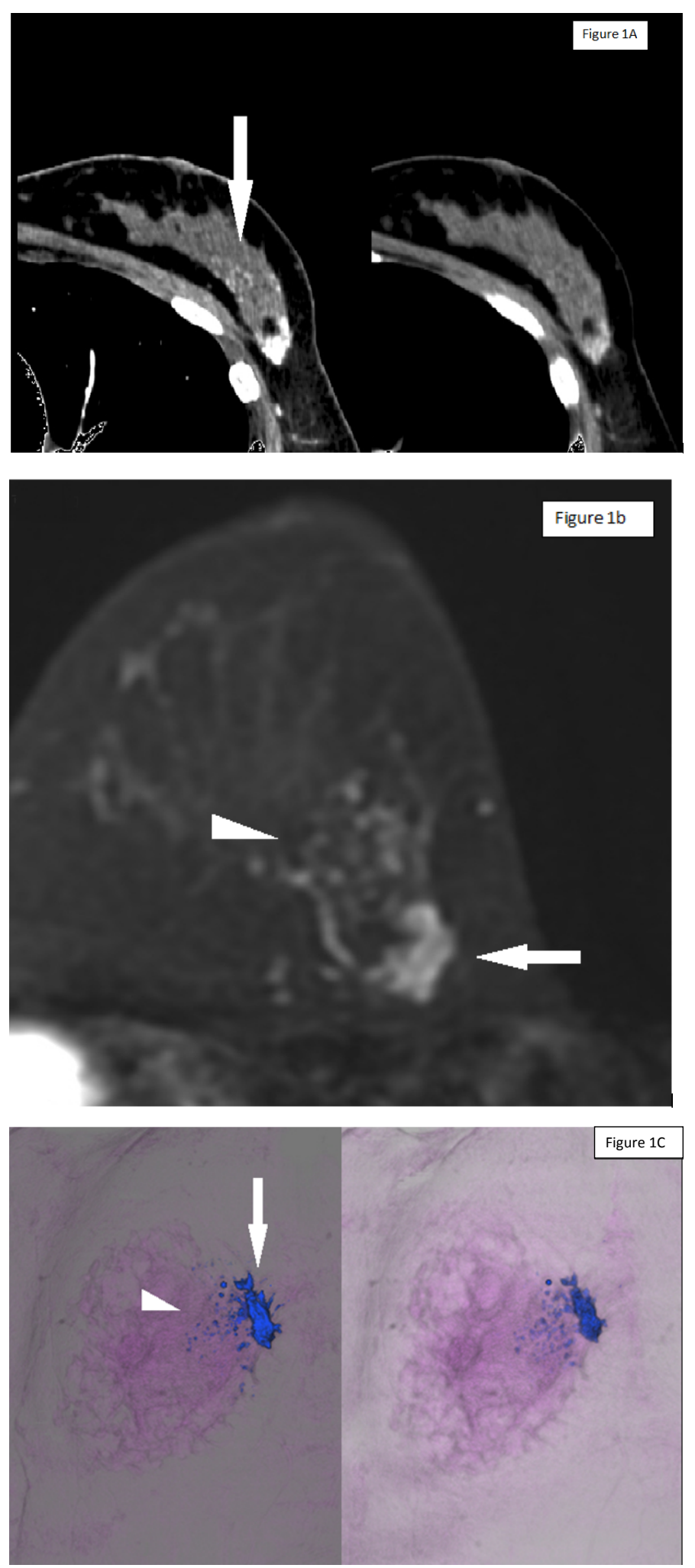

Figure 1. Axial images of breast cancer and intraductal components (ICs): segmental distribution type. (A) Post-contrast dual-energy CT (DECT) images comparison of $40 \mathrm{keV}$ (left) and 65keV (right) images of same window and level. ICs (arrow) are clearly visualized on $40 \mathrm{keV}$ image with high contrast. (B) Original 3D-MIP images of post-contrast MRI (1.5T) shows ICs (arrowhead) in extended breast as the patient was in a prone position. Positional relation of ICs and main tumour (arrow) is visualized. (C) Oblique virtual rendering (VR) images of Dual-energy CT (DECT), comparison of $40 \mathrm{keV}$ (left) and $65 \mathrm{keV}$ (right) images. Intraductal components (ICs) of segmental distribution type are identified clearly on $40 \mathrm{keV}$ image (arrowhead). 
pre-surgical staging were performed in $65 \mathrm{keV}$ axial images with a slice thickness of $5 \mathrm{~mm}$. For MRI, IC was evaluated and primary tumour spread was assessed mainly in a dynamic study and in original 3D-MIP images (FIGURES 1B and $2 \mathrm{~B}$ ) and diffusion images.

Results were compared by the chi-squared test and the $P$ value was cited. Statistical analyses were performed using statistical software (Microsoft Excel 2013, Microsoft, Redmond, WA, USA) with the StatFlex version 6.0 add-in (Artech Co., Ltd, Osaka, Japan).

\section{Results}

The results of the correlation between pathologically proven ICs and ICs judgement by DECT or MRI are presented in TABLE 2. Pathologically, an IC of breast cancer was identified in 26 patients. On DECT, an IC was identified in 32 patients; however, the findings in 12 of these patients were false positive. On MRI, an IC was identified in 35 patients; however, the findings in 16 of these patients were false positive. The sensitivity, specificity and accuracy for detecting an IC of breast cancer were $76.9 \%$, 58.6\% and 67.3\% $(P=0.008)$ with DECT and were $73.1 \%, 44.8 \%$ and $58.2 \%(P=0.043)$ with MRI, respectively. The detection of the presence of an IC on DECT was highly correlated with the pathological outcome, and DECT indicated the presence of an IC with higher sensitivity when compared with that for MRI.

\section{Discussion}

The clinical utility of DECT has been rapidly progressing, as demonstrated by the high number of articles published during the past years. Several studies have reported the utility of DECT for detecting breast cancer and an IC $[18,19]$, as virtual low-monochromatic images of dual-energy scanning provide improved contrast resolution in breast tissue when compared with that with SECT images.

Dual-energy scanning involves imaging at high and low $\mathrm{kV}$ values, providing differing attenuation data sets from which an image can be reconstructed. Typically, materials, such as calcium, iodine and water, possess sufficiently different linear attenuations at low and high energies to allow for clear discrimination. However, materials with very similar attenuation curves, such as soft tissue, fat and water, can cause problems, and they require advanced analytical techniques, such as beam pre-filtering or three-compartment analysis at a high $\mathrm{kV}$. It is significant that the greatest attenuation differences typically exist at lower energies, and this allows contrast differentiation in the lower range of attenuation. This fact is compatible with the results of studies showing that low keV DECT images have a high contrast enhancement effect with high resolution in soft tissues [17,22,23]. Breast cancer and surrounding normal breast tissue have soft tissue with relatively similar attenuation; thus, in this study, assessments were attempted in reconstructed images at a low $\mathrm{keV}$. DECT data calculation has been utilized to improve image contrast and increase the intravascular iodine signal in poor contrast conditions, which can reduce contrast material volume in contrastenhanced CT [30-32]. We did not attempt to reduce contrast material volume in this study. However, the possibility of reducing contrast material volume should be assessed as it could be valuable in patients with insufficient renal function.

We compared the visualization of an IC between 40 and $65 \mathrm{keV}$ images reconstructed with a slice thickness of $1 \mathrm{~mm}$ and found that $40 \mathrm{keV}$ images were more appropriate for visualizing an IC, as fine structures were more distinct in $40 \mathrm{keV}$ images. The contrast ratio in $40 \mathrm{keV}$ DECT images enables differentiation between a contrast-enhanced IC and normal breast tissue through analysis of the minimal difference of attenuation. Thus, we could draw the detailed shapes of ICs in VR images (FIGURES 3 and 4). We believe that $40 \mathrm{keV}$ DECT images can add more detailed and important diagnostic information to the findings of $65 \mathrm{keV}$ DECT images and contrast-enhanced MRI images.

Considering that oncological patients undergo repeated follow-up CT examinations, dose issues should not be neglected and the management of overall radiation exposure should be considered important. Recent studies have mentioned that DECT can be performed without radiation dose penalty or reduction in image quality when compared with SECT [33-35]. Optimization of the radiation dose in CT is indispensable [35], however, imaging quality should not be affected. In our study, noise and the radiologist's perception of DECT image quality were clinically acceptability. Furthermore, IC 

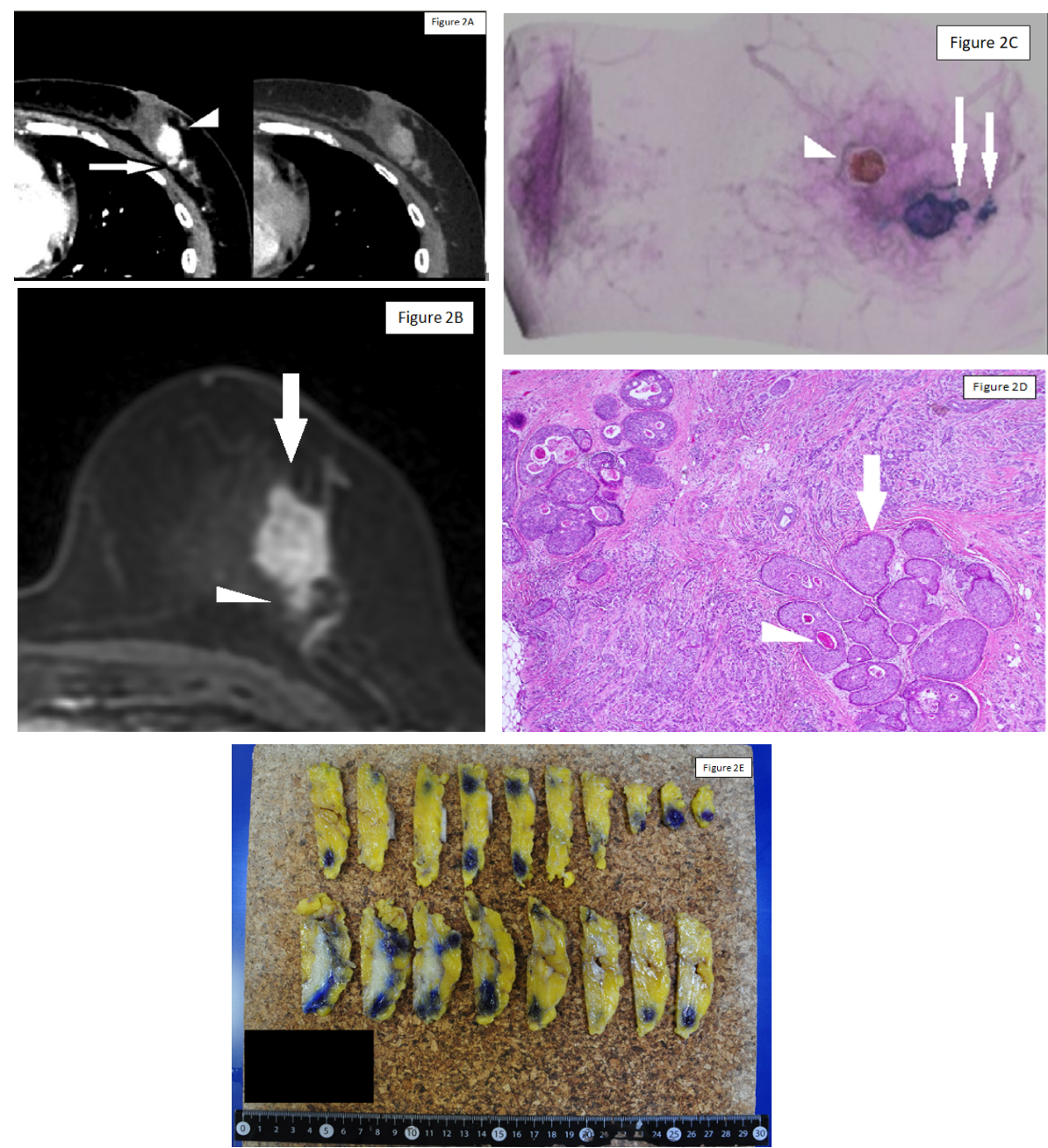

Figure 2. Axial images of breast cancer and intraductal components (ICs): ductal extension type. (A) Post-contrast DECT images of ICs of $40 \mathrm{keV}$ (left) and $65 \mathrm{keV}$ (right) of same window and level. ICs (arrow) arising from the breast cancer (arrowhead) is visualized with high contrast on $40 \mathrm{keV}$ image. (B) Original 3D-MIP images of post-contrast MRI (1.5T) shows an IC (arrowhead) arising from the main tumour (arrow). (C) Oblique virtual rendering (VR) image of Dual-energy CT (DECT) reconstructed by $40 \mathrm{keV}$. Intraductal components (ICS) of ductal extension type are identified (arrows). (arrowhead: fibroadenoma). (D) Pathological finding of the same case. At high magnification, the malignant cells are confined to the ducts (arrow), which are dilated. Malignant cells do not invade either the mammary fibrous stroma or fat. Note intraductal component with papillary pattern and microcalcifications (arrowhead). Hematoxylin and eosin stain. (E) Sliced surgical specimen after marking of intraductal components. An attempt to visualize threedimensional situation of intraductal components in the specimen was performed.

\begin{tabular}{|c|c|c|c|c|c|c|c|}
\hline & Accuracy & Sensitivity & Specificity & $\begin{array}{l}\text { Positive } \\
\text { predictive } \\
\text { value }\end{array}$ & $\begin{array}{c}\text { Number of } \\
\text { positive cases } \\
\text { (false positive) }\end{array}$ & $\begin{array}{c}\text { Number of negative } \\
\text { cases (false } \\
\text { negative) }\end{array}$ & $P$ value \\
\hline DECT positive & $\begin{array}{l}67.3 \% \\
(38 / 55)\end{array}$ & $\begin{array}{l}76.9 \% \\
(20 / 26)\end{array}$ & $\begin{array}{l}58.6 \% \\
(17 / 29)\end{array}$ & $\begin{array}{l}68.4 \% \\
(26 / 38)\end{array}$ & $32(12)$ & $23(6)$ & 0.008 \\
\hline MRI positive & $\begin{array}{l}58.2 \% \\
(32 / 55)\end{array}$ & $\begin{array}{l}73.1 \% \\
(19 / 26)\end{array}$ & $\begin{array}{l}44.8 \% \\
(13 / 29)\end{array}$ & $\begin{array}{l}61.9 \% \\
(26 / 42)\end{array}$ & $35(16)$ & $20(7)$ & 0.043 \\
\hline $\begin{array}{l}\text { DECT \& MRI } \\
\text { positive }\end{array}$ & $\begin{array}{l}67.2 \% \\
(38 / 55)\end{array}$ & $\begin{array}{l}69.0 \% \\
(18 / 26)\end{array}$ & $\begin{array}{l}58.6 \% \\
(17 / 29)\end{array}$ & $\begin{array}{l}68.4 \% \\
(26 / 38)\end{array}$ & $30(12)$ & $15(8)$ & 0.038 \\
\hline
\end{tabular}

visualization and pre-operative staging were performed simultaneously on DECT without an additional radiation dose.
The presence of an IC has been mainly assessed on contrast-enhanced MRI because of its high contrast ratio. However, its spatial resolution is relatively insufficient, especially with a system of 


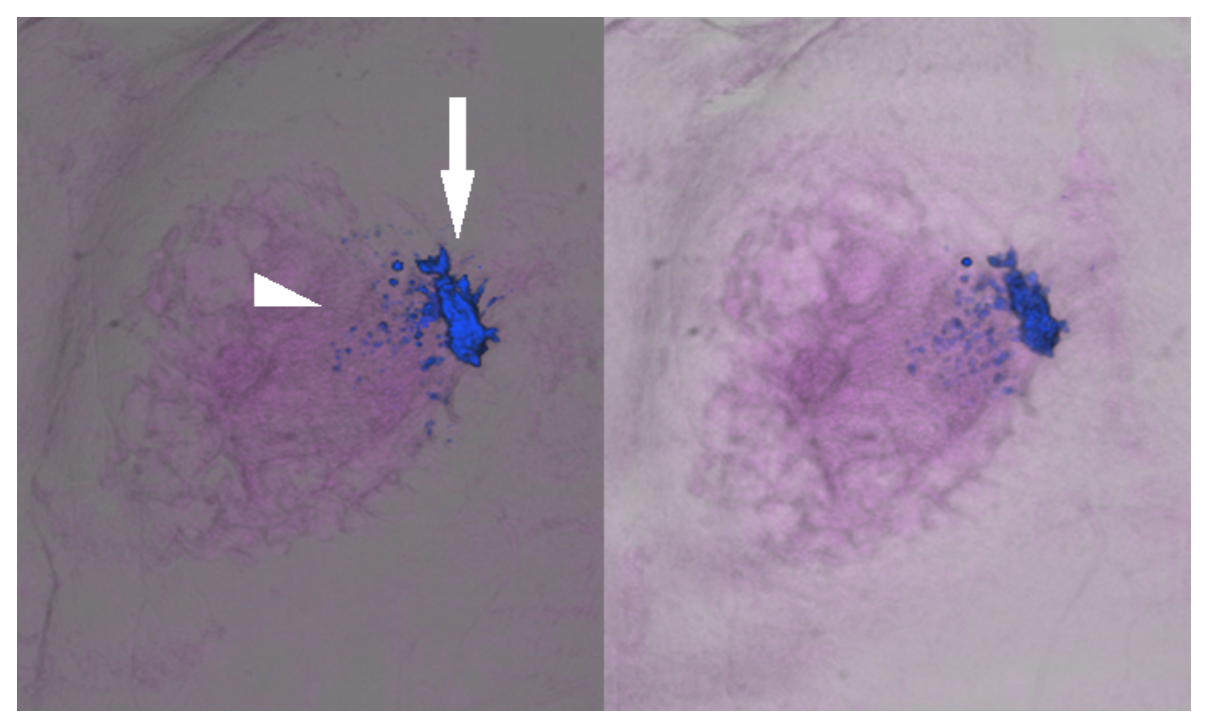

Figure 3. Oblique virtual rendering (VR) images of Dual-energy CT (DECT), comparison of $40 \mathrm{keV}$ (left) and $65 \mathrm{keV}$ (right) images. Intraductal components (ICs) of segmental distribution type are identified clearly on $40 \mathrm{keV}$ image (arrowhead).

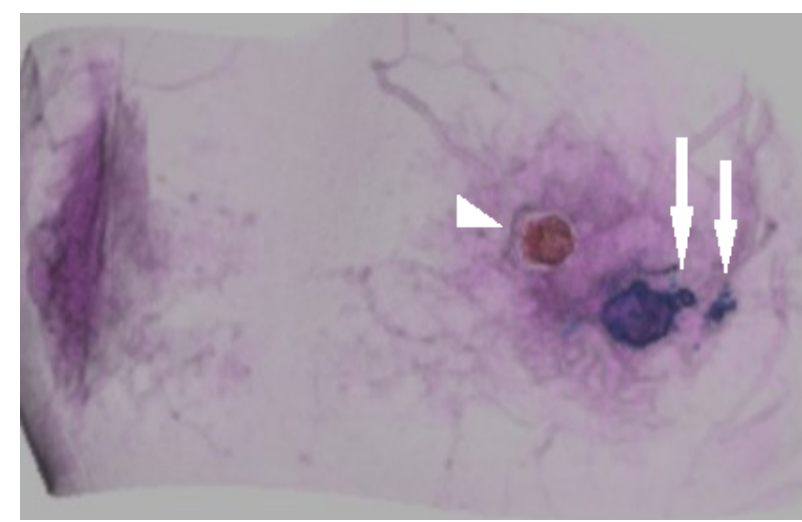

Figure 4. Oblique virtual rendering (VR) image of Dual-energy CT (DECT) reconstructed by $40 \mathrm{keV}$. Intraductal components (ICS) of ductal extension type are identified (arrows). (arrowhead: fibroadenoma).

1.5T or less, and it might be difficult to use these MRI images for surgical support considering the shape of the breast receiver coil and the face-down position adopted. An advantage of DECT is the ability to perform imaging in the face-up position, which is consistent with the surgical position. Furthermore, DECT can be considered useful for pre-surgical assessment to determine the appropriate surgical procedure in patients who are contraindicated for MRI, such as those who have claustrophobia and those who have a non-MRI conditional cardiac pacemaker.

The present study had some limitations. First, this was a retrospective study, and the number of patients was relatively small. Thus, research to evaluate the utility and reliability of DECT as a pre-operative examination should be continued. In this study, we focused on the diagnostic detection of an IC, as the number of patients was small. Studies with a larger number of patients and detailed parameters, such as histological characteristics and disparity of imaging configurations, might provide advanced results. Second, we compared the data sets of 1.5T MRI with those of DECT in this study, although $3 \mathrm{~T}$ MRI systems are utilized for investigating breast cancer in many facilities. The 3T MRI system is superior to the $1.5 \mathrm{~T}$ MRI system with regard to the identification of an IC and the contribution to the histological diagnosis of breast cancer. Thus, the results might be different with a $3 \mathrm{~T}$ MRI system. Third, we focused on the presence of an IC in DECT and MRI images regardless of histological differences, as the number of patients was small. A comparative study considering histopathological classification should be performed, as the presence and 
presentation of an IC can vary depending on histological differences. Furthermore, detailed measurement, configurational IC evaluation and distinction between ductal extension and segmental distribution might be useful as preoperative assessments. Fourth, we used $40 \mathrm{keV}$ images for assessing the presence of an IC and $65 \mathrm{keV}$ images for assessing distal metastasis, as a substitution for usual contrast-enhanced CT images. However, assessment of images reconstructed with other parameters was not attempted in this study. Other reconstruction parameters might improve the visualization of fine structures, such as an IC and might improve the statistical results.

\section{Conclusions}

DECT is a useful supporting modality for the detection of an IC of breast cancer and is a reliable additional approach. The advantages of DECT include high detectability of an IC (highly correlated with the pathological outcome), clinically acceptable image quality, imaging in the face-up position (consistent with the surgical position) and simultaneous assessment of distant metastasis without radiation dose penalty. Furthermore, DECT can be used for the pre-surgical assessment of an IC in patients who are contraindicated for MRI, and this will help in the selection of the most appropriate surgical procedure.

\section{REFERENCES}

1. Rashid OM, Nagahashi M, Ramachandran S et al. Resection of the primary tumor improves survival in metastatic breast cancer by reducing overall tumor burden. Surgery. 153, 771-778 (2013).

2. Bafford AC, Burstein HJ, Barkley CR et al. Breast surgery in stage IV breast cancer: impact of staging and patient selection on overall survival. Breast. Cancer. Res. Treat. 115,7-12 (2009).

3. Rapiti E, Verkooijen HM, Fioretta G et al. Complete excision of primary breast tumor improves survival of patients with metastatic breast cancer at diagnosis. J. Clin. Oncol. 20, 2743-2749 (2006).

4. Leung AM, Vu HN, Nguyen KA et al. Effects of surgical excision on survival of patients with stage IV breast cancer. J. Surg. Res. 161, 83-88 (2010).

5. Lieberman L, Morris EA, Dershaw DD et al. Ductal enhancement on MR imaging of the breast. AJR. 181, 519-525 (2003).

6. Siegmann KC, Muller SM, Schlick F et al. MR imaging-detected breast lesions: histopathologic correlation of lesion characteristics and signal intensity data. AJR. 178, 1403-1409 (2002).

7. Mumtaz H, Hall CMA, Davidson $\mathrm{T}$ et al. Staging of symptomatic primary breast cancer with MR imaging. AJR. 169, 417-424 (1997).

8. Ishida T, Furuta A, Moriya T et al. Pathological assessment of intraducutal spread of carcinoma in relation to surgical margin state in breastconserving surgery. Jap. J. Clin. Oncol. 33, 161166 (2003).

9. Hurd TC, Steiger N, Allen PK et al. Impact of extensive intraductal component on recurrence and survival in patients with stage 1 or 2 breast cancer treated with breast conservation therapy. Annals. Surg. Oncol. 4, 119-124 (1997).
10. Holland R, Connolly JL, German R et al. The presence of an extensive intraductal component following a limited excision correlates with prominent residual disease in the remainder of the breast. J. Clin. Oncol. 8, 113-118 (1990).

11. Shimauchi A, Yamada T, Sato A et al. Comparison of MDCT and MRI for evaluating the intraductal component of breast cancer. AJR. 187, 322-329 (2006)

12. Kim H, Jung H, Ko K et al. Mammography, US, and MRI for preoperative prediction of extensive intraductal component of invasive breast cancer: Interobserver variability and performances. Breast. Cancer. 16, 305-311 (2016).

13. Menezes GLG, Knuttel FM, Stehouwer BL et al. Magnetic resonance imaging in breast cancer: A literature review and future perspectives World. J. Clin. Oncol. 10, 61-70 (2014).

14. Wisner DJ, Hwang ES, Chang CB et al. Features of occult invasion in biopsy-proven DCIS at breast MRI. Breast. J. 19, 650-658, (2013).

15. Johnson TRC. Dual-energy CT: general principles. AJR. 119, 3-8 (2012).

16. Wichmann JL, Hardie AD, Schoepf UJ et al. Single- and dual-energy CT of the abdomen: comparison of radiation dose and image quality of 2 nd and 3rd generation dual-source CT. Eur. Radiol. 27, 642-650 (2017).

17. Mileto A, Sofue K, Marin D. Imaging the renal lesion with dual-energy multidetector CT and multi-energy applications in clinical practice: what can it truly do for you?. Eur. Radiol. 26, 3677-3690 (2016).

18. Heye T, Nelson RC, Ho LM et al. Dual energy CT Applications in the Abdomen. AJR. 199, 64-70 (2012).

19. Tang CX, Zhou CS, Zhao YE et al. Detection of pulmonary fat embolism with dual-energy CT: an experimental study in rabbits. Eur. Radiol.

\section{7, 1377-1385 (2017).}

20. Otrakji A, Digumarthy SR, Lo Gullo R et al. Dualenergy CT: Spectrum of thoracic abnormalities. RadioGraphics. 36, 38-52 (2016).

21. DC Cecco, A Darnell, N Macias et al. Secondgeneration dual-energy computed tomography of the abdomen radiation dose comparison with 64- and 128-row single-energy acquisition. J. Comput. Assist. Tomogr. 37, 543-546 (2013).

22. Grajo JR, Patino M, Prochowski A et al. Dual energy CT in practice: Basic principles and applications. Appl. Radiol. 45, 6-12 (2016).

23. Finkenstaedt T, Manoliou A, Toniolo M et al. Gouty arthritis: the diagnostic and therapeutic impact of dual-energy CT. Eur. Radiol. 26, 3989-3999 (2016).

24. Okamura Y, Yoshizawa N, Yamaguchi M et al. Application of dual-energy computed tomography for breast cancer diagnosis. Int. J. Med. Phys. Clin. Eng. Radiat. Oncol. 5, 288-297 (2016).

25. Le H, Ducote J, Molloi S. Dual energy contrastenhanced breast computed tomography: A phantom Study. Med. Phys. 35, 2978 (2008).

26. Glazebrook KN, Leng S, Jacobson SR et al. Case report dual-energy CT for evaluation of intra and extracapsular silicone implant rupture. Case. Reports. Radiology. 16, 1-4 (2016)

27. Ding H, Ducote JL, Molloi S. Measurement of breast tissue composition with dual energy conebeam computed tomography: A postmortem study. Med. Phys 40, 1-9 (2013).

28. Noid G, Currey AD, Bergom C et al. Improvement of breast tumor delineation for Preoperative radiation therapy using dualenergy CT. Int. J. Radiat. Oncol. Biol. Phys. 99, 704 (2017).

29. Johnson TRC, Himsl I, Hellerhoff K et al. Dualenergy CT for the evaluation of silicone breast 
implants. Eur. Radiol. 23, 991-996 (2013).

30. McCollough $\mathrm{CH}$, Leng $\mathrm{S}$, Yu L et al. Dual and multi-energy CT: Principles, technical approaches, and clinical applications. Radiology. 276, 637-653 (2015)

31. Mileto A, Ramirezgiraldo JC, Marin D et al. Nonlinear image blending for dual-energy MDCT of the abdomen: Can image quality be preserved if the contrast medium dose is reduced?. AJR. 203, 838-845 (2014).

32. Mileto A, Ramirezgiraldo JC, Marin D et al. Dual-energy MDCT of the preserved if the contrast medium. AJR. 13, 1-5 (2014).

33. Uhrig $\mathrm{M}$, Simons $\mathrm{D}$, Kachelrie $\mathrm{M}$ et al. Advanced abdominal imaging with dual energy $\mathrm{CT}$ is feasible without increasing radiation dose. Cancer. Img. 16, 1-8 (2016).
34. Albrecht MH, Scholtz JE, Husers $\mathrm{K}$ et al. Advanced image-based virtual monoenergetic dual-energy CT angiography of the abdomen: optimization of kilo electron volt settings to improve image contrast. Eur. Radiol. 26, 1863 1870 (2016).

35. Tsushima Y, Taketomi TA, Takei $\mathrm{H}$ et al. Radiation Exposure from CT Examinations in Japan. BMC. Med. Imaging. 10, 1-8 (2010). 